\title{
A Short Proof of an Interesting Helly-Type Theorem*
}

\author{
N. Amenta \\ The Geometry Center, 1300 South Second Street, \\ Minneapolis, MN 55454, USA \\ nina@geom.umn.edu
}

\begin{abstract}
We give a short proof of the theorem that any family of subsets of $R^{d}$, with the property that the intersection of any nonempty finite subfamily can be represented as the disjoint union of at most $k$ closed convex sets, has Helly number at most $k(d+1)$.
\end{abstract}

\section{Introduction}

We say that a family of sets $\mathcal{F}$ has Helly number $h$ when $h$ is the smallest integer (if one exists) such that any finite subfamily $\mathcal{H} \subseteq \mathcal{F}$ has nonempty intersection if and only if every subfamily $\mathcal{B} \subseteq \mathcal{H}$ with $|\mathcal{B}| \leq h$ also has nonempty intersection. Theorems of the form " $\mathcal{F}$ has Helly number $h$ " are called Helly-type theorems-they follow the model of Helly's theorem, which states that the family of convex sets in $R^{d}$ has Helly number $d+1$. There are many Helly-type theorems; for excellent surveys see [DGK] and the recent [E].

This paper is concerned with a generalization of Helly's theorem:

Theorem 1.1. Let $\mathcal{F}$ be a family of sets in $R^{d}$, such that the common intersection of any nonempty finite subfamily of $\mathcal{F}$ can be expressed as the disjoint union of at most $k$ closed convex sets. Then $\mathcal{F}$ has Helly number at most $k(d+1)$.

Note that when the common intersection of any at most $k$ members of $\mathcal{F}$ can be expressed as the disjoint union of at most $k$ closed convex sets, so can the intersection of any finite subfamily [GM, Theorem 2].

Theorem 1.1 was first conjectured by Grünbaum and Motzkin in 1961 [GM]. They

* Some of this work was done at the University of California, Berkeley, where the author was supported by a U.C. Presidents Dissertation Year Fellowship and an AT\&T Graduate Research Program for Women Grant. 
proved the case $k=2$, using a more general axiomatic structure in place of convexity. Rather than convex sets, their theorem applies to any set family $\mathcal{C}$ with Helly number $d+1$ for which the intersection of any pair of sets in $\mathcal{C}$ is again a member of $\mathcal{C}$, and for which the disjoint union of any pair of sets in $\mathcal{C}$ is not a member of $\mathcal{C}$. Larman proved the case $k=3$ [L], for convex sets. Morris treated the question in his thesis [M], again using a combinatorial generalization of convexity. The proof he offered, however, is very long and involved, and its correctness is questionable (see p. 399 of [E]). Some related results appear in [HT].

We give a short and intuitive proof, using a different axiomatic system, borrowed from computational geometry. Our approach is to introduce an ordering $\preceq$ on the points of $R^{d}$, and study the problem of minimizing $\preceq$ over any subfamily of $\mathcal{F}$. We show that this problem is an example of an LP-type (or Generalized Linear Programming, or GLP) problem. The theorem follows from the observation that there is a Helly-type theorem about the constraint set of every LP-type problem.

Informally, the LP-type problems are the class of problems which can be solved by combinatorial linear programming algorithms such as those given in $[\mathrm{S}],[\mathrm{C}]$, and [MSW]. So the minimization problem we construct is computationally similar to linear programming, although geometrically the intersection of the constraints fails not only to be convex, but even to be connected. This suggests the possibility of applying linear programming algorithms to other problems in which the the topological complexity of the intersection of the constraints, although nontrivial, remains bounded by a constant.

\section{Framework}

LP-type problems are defined by an abstract combinatorial framework due to Sharir and Welzl [SW]. We use a slightly less abstract definition for a subclass of LP-type problems, which we call concrete LP-type problems.

Consider a triple $(W, \mathcal{H}, \preceq$ ), where the universe $W$ is a set (whose elements we call points), $\mathcal{H}$ is a finite family of subsets of $W$ (called the constraints), and $\preceq$ is an order on $W$, with the symbol $+\infty$ defined to be $\succ$ any point of $W$. For any subfamily of constraints $\mathcal{G} \subseteq \mathcal{H}$, we denote the intersection $\bigcap \mathcal{G}=\{x \in W \mid x \in G, \forall G \in \mathcal{G}\}$

$(W, \mathcal{H}, \preceq$ ) is a concrete $L P$-type problem if, for every $\mathcal{G} \subseteq \mathcal{H}$ with $\bigcap \mathcal{G}$ nonempty, $\bigcap \mathcal{G}$ has a unique minimum point; we call this point $w(\mathcal{G})$ and we say that $\mathcal{G}$ is feasible. When $\bigcap \mathcal{G}$ is empty we say that $\mathcal{G}$ is infeasible and we define $w(\mathcal{G})=+\infty$.

The relevant example, for our purposes, of a concrete LP-type problem is Lexicographic Convex Programming, in which $W$ is $R^{d}, \mathcal{H}$ is any finite family of compact convex sets, and $\preceq$ is the lexicographic order on $R^{d}$. Another example is Normal Convex Programming, in which $W$ again is $R^{d}, \mathcal{H}$ again is any finite family of compact convex sets, and $\preceq$ orders points by their distance from the origin. Notice that in Normal Convex Programming, $\preceq$ is not a total order on $R^{d}$, although every subfamily of constraints does have a unique minimum point.

The interested reader can easily verify that for every concrete LP-type problem $(W, \mathcal{H}, \preceq)$, the pair $(\mathcal{H}, w)$ is an LP-type problem as defined in [SW] or [MSW].

A basis is a subfamily $\mathcal{G} \subseteq \mathcal{H}$ such that $w(\mathcal{G}-G) \prec w(\mathcal{G})$, for all $G \in \mathcal{G}$. The 
combinatorial dimension of a concrete LP-type problem is the maximum cardinality of any feasible basis. The combinatorial dimension, for example, of Lexicographic Convex Programming in $R^{d}$ is $d$. It is not difficult to see that every subfamily $\mathcal{G}$ must contain a basis $\mathcal{B} \subseteq \mathcal{G}$ with $w(\mathcal{B})=w(\mathcal{G})$, and that for three subfamilies $\mathcal{B} \subseteq \mathcal{F} \subseteq \mathcal{G}$ with $w(\mathcal{B})=w(\mathcal{G})$, it must also be the case that $w(\mathcal{F})=w(\mathcal{B})$. (See [SW] or [MSW] for other simple properties of LP-type problems.)

Lemma 2.1. Let $(W, \mathcal{H}, \preceq)$ be a concrete LP-type problem of combinatorial dimension c. $\mathcal{H}$ has Helly number at most $c+1$.

Proof. $\mathcal{H}$ has Helly number at most $h$ if and only if every subfamily $\mathcal{G}$ with $\cap \mathcal{G}$ empty contains a subfamily $\mathcal{B} \subseteq \mathcal{G}$ with $\cap \mathcal{B}$ empty and $|\mathcal{B}| \leq h$. The Helly number, then, is the maximum cardinality of any infeasible basis, while the combinatorial dimension is the maximum cardinality of any feasible basis.

So let $\mathcal{B}$ be any infeasible basis, and let $G \in \mathcal{B}$ be any constraint with $w(\mathcal{B}-G) \approx$ $\max _{\leq}\left\{w\left(\mathcal{B}-G^{\prime}\right) \mid G^{\prime} \in \mathcal{B}\right\}$, under $\preceq$. The subfamily $\mathcal{B}-G$ is feasible and contains a basis $\mathcal{B}^{\prime}$ with $\left|\mathcal{B}^{\prime}\right| \leq c$ and the point $w\left(\mathcal{B}^{\prime}\right)=w(\mathcal{B}-G)$. We show that every constraint $G^{\prime} \in \mathcal{B}-G$ must in fact belong to $\mathcal{B}^{\prime}$.

Assume the contrary, that is, $\mathcal{B}^{\prime} \subset \mathcal{B}-G-G^{\prime}$ for some such $G^{\prime}$. Then $w\left(\mathcal{B}^{\prime}\right) \preceq$ $w\left(\mathcal{B}-G-G^{\prime}\right)$. If $w\left(\mathcal{B}-G^{\prime}\right) \prec w(\mathcal{B}-G)$, then $w\left(\mathcal{B}-G-G^{\prime}\right) \preceq w\left(\mathcal{B}-G^{\prime}\right) \prec w(\mathcal{B}-G)=$ $w\left(\mathcal{B}^{\prime}\right)$, a contradiction. Otherwise, $w\left(\mathcal{B}-G^{\prime}\right) \approx w(\mathcal{B}-\bar{G})$, that is, the points are equivalent under $\preceq$ although they are not necessarily identical. The point $w(\mathcal{B}-G) \in G^{\prime}$, whereas the infeasibility of $\mathcal{B}$ implies that $w\left(\mathcal{B}-G^{\prime}\right) \notin G^{\prime}$. Since the minimum of $w\left(\mathcal{B}-G-G^{\prime}\right)$ must be achieved at a unique point, we have $w\left(\mathcal{B}-G-G^{\prime}\right) \prec w\left(\mathcal{B}-G^{\prime}\right) \approx$ $w(\mathcal{B}-G)=w\left(\mathcal{B}^{\prime}\right)$, again a contradiction. We conclude that $\mathcal{B}-G=\mathcal{B}^{\prime}$ and so $|\mathcal{B}|=\left|\mathcal{B}^{\prime}\right|+1 \leq c+1$.

\section{Main Theorem}

Theorem 3.1. Let $(W, \mathcal{C}, \preceq)$ be a concrete LP-type problem of combinatorial dimension $d$ with the additional property that $\preceq$ is a total order on the points of $W$. Let $\mathcal{H}$ be a family of subsets of $W$ such that, for every $\mathcal{G} \subseteq \mathcal{H}$ with $\bigcap \mathcal{G} \neq \emptyset, \bigcap \mathcal{G}$ can be written as the disjoint union of at most $k$ elements of $\mathcal{C}$. Then $(W, \mathcal{H}, \preceq)$ is an LP-type problem of combinatorial dimension at most $k(d+1)-1$.

We need some notation for working with disjoint unions. Our assumption is that $\bigcap \mathcal{G}$ can be written as the union of disjoint sets $c_{1}, c_{2}, \ldots \in \mathcal{C}$, which we call the components of $\cap \mathcal{G}$. Consider some point $p \in W$. For each individual $G \in \mathcal{G}, p$ is contained in at most one component $c(G, p)$ of $G$. Let $C(\mathcal{G}, p)=\{c(G, p) \mid G \in \mathcal{G}\}$, that is, the collection of the components from the individual constraints containing the point $p$. If $p \in \bigcap \mathcal{G}$, the component $c(\bigcap \mathcal{G}, p)$ of $\bigcap \mathcal{G}$ containing $p$ is exactly $\cap C(\mathcal{G}, p)$.

Proof of Theorem 3.1. Because $\preceq$ is a total order, the minimum of $\preceq$ over any intersection $\bigcap \mathcal{G}, \mathcal{G} \subseteq \mathcal{H}$, is achieved at a unique point, and $(W, \mathcal{H}, \preceq)$ is a concrete LP-type 
problem. It remains to show that the maximum size of any feasible basis $\mathcal{B}$ is at most $k(d+1)-1$.

We count the constraints in a feasible basis $\mathcal{B}$ by carefully removing selected constraints, one at each step, while building up a subfamily $\mathcal{S}$ of "sacred" constraints which may not be removed in later steps. After step $t$ we call the current sets $\mathcal{S}_{t}$ and $\mathcal{B}_{t}$, and we call the minimum point $w_{\imath}=w\left(\mathcal{B}_{t}\right)$.

We maintain two invariants. The first is that $w\left(\mathcal{B}_{t}-G\right) \prec w_{t}$ for all $G \in \mathcal{B}_{t}-\mathcal{S}_{t}$. The second is that all the points $w_{1}, w_{2}, \ldots, w_{t}$ lie in different components of $\cap \mathcal{B}_{t}$.

We set $t=1$ and $\mathcal{B}_{1}=\mathcal{B}$. Notice that since $\mathcal{B}$ is a basis, the first invariant will hold for any initial choice of $\mathcal{S}_{1}$. We choose $\mathcal{S}_{1}$ so as to guarantee that $w_{1}$ lies in a different component from all other $w_{i}$ during all future steps. We start with $\mathcal{S}_{0}=\emptyset$, and use the following general procedure, applicable at any step $t$, for adding constraints to $\mathcal{S}_{t-1}$ to get $\mathcal{S}_{t}$.

Since $\bigcap \mathcal{B}_{t}$ is nonempty, there is a unique minimum point $w_{t}=w\left(\mathcal{B}_{t}\right)$ in $\bigcap \mathcal{B}_{t}$. Let $C_{t}=C\left(\mathcal{B}_{t}, w_{t}\right)$, that is, the collection of components from the individual constraints containing $w_{t}$. Notice that $\left(W, C_{t}, \preceq\right)$ is a feasible instance of the given concrete LP-type problem of combinatorial dimension $d$, with $w\left(C_{t}\right)=w_{t}$. So $C_{t}$ must contain a basis $\mathcal{B}_{t}^{\prime}$ with $\left|\mathcal{B}_{t}^{\prime}\right| \preceq d$ and $w\left(\mathcal{B}_{t}^{\prime}\right)=w_{t}$. For each $c \in \mathcal{B}_{t}^{\prime}$, select a constraint $G \in \mathcal{B}_{t}$ having $c$ as a component, and let $\mathcal{A}_{t}$ be the family of these constraints. We set $\mathcal{S}_{l}=\mathcal{S}_{t-1} \cup \mathcal{A}_{t}$.

This procedure guarantees that the second invariant is preserved. Consider the situation at some step $t$. The current collection of components containing the point $w_{i}$, for any $t>i \geq 1$, is $C\left(\mathcal{B}_{t}, w_{i}\right)$. Since $\mathcal{A}_{i} \subseteq \mathcal{S}_{t} \subseteq \mathcal{B}_{t}, C\left(\mathcal{B}_{t}, w_{i}\right)$ still contains the basis $\mathcal{B}_{i}^{\prime}$, which means that $w_{i}$ must still be the minimum point in $\bigcap C\left(\mathcal{B}_{l}, w_{i}\right)$. Since $w_{j} \prec w_{i}$ for $t \geq j>i$, each point $w_{j}$ must lie in some component other than $\bigcap C\left(\mathcal{B}_{t}, w_{i}\right)$. This forces all the components $c\left(\bigcap \mathcal{B}_{t}, w_{i}\right)$ to be distinct.

Now we turn our attention to selecting a constraint to remove from $\mathcal{B}_{t}$ to create $\mathcal{B}_{t+1}$. First notice that the points $w\left(\mathcal{B}_{t}-G\right)$ are distinct, for all $G \in \mathcal{B}_{t}-\mathcal{S}_{t}$. Indeed, let $G, G^{\prime} \in \mathcal{B}_{t}-\mathcal{S}_{t}$ be distinct constraints. Then $w\left(\mathcal{B}_{t}-G^{\prime}\right) \in G$, while $w\left(\mathcal{B}_{t}-G\right) \notin G$ since $w\left(\mathcal{B}_{t}-G\right) \prec w\left(\mathcal{B}_{t}\right)$. The points of $W$ are totally ordered under $\preceq$, so there is some $G_{t} \in \mathcal{B}_{t}-\mathcal{S}_{t}$ such that $w\left(\mathcal{B}_{t}-G\right) \prec w\left(\mathcal{B}_{t}-G_{t}\right)$ for all other $G \in \mathcal{B}_{t}-\mathcal{S}_{t}$.

It is $G_{t}$ that we remove from $\mathcal{B}_{t}$ to form $\mathcal{B}_{t+1}$. Since $w\left(\mathcal{B}_{t}-G\right) \prec w\left(\mathcal{B}_{t}-G_{t}\right)$, for all other $G \in \mathcal{B}_{t}-\mathcal{S}_{t}$, certainly $w\left(\mathcal{B}_{t}-G_{t}-G\right) \prec w\left(\mathcal{B}_{t}-G_{t}\right)$. So the first invariant is maintained for $\mathcal{B}_{t+1}$. To ensure the preservation of the second invariant, we again follow the procedure above to find a set $\mathcal{A}_{t+1}$ to add to $\mathcal{S}_{t}$ to get $\mathcal{S}_{t+1}$.

We iterate this process of removing a constraint from $\mathcal{B}_{t}$ and updating $\mathcal{S}_{t}$ until we can no longer continue because $\mathcal{B}_{t}=\mathcal{S}_{t}$.

The common intersection of any subfamily of constraints can be described as the disjoint union of at most $k$ components, so at the end of the process there are at most $k$ points $w_{i}$ and the number of steps is $t \leq k$. We removed one constraint $G_{i}$ at every step except for the first, and at every step, we added at most $d$ constraints to $\mathcal{S}_{i}$. So the size of $|\mathcal{B}|$ is at most $(k-1)+k d=k(d+1)-1$.

Theorem 1.1 is an easy application of Theorem 3.1.

Proof of Theorem 1.1. We take Lexicographic Convex Programming as the concrete LP-type problem $(W, \mathcal{C}, \preceq)$ in Theorem 3.1. 
Since the constraints of a lexicographic convex program have to be compact, we construct for any nonempty finite subfamily $\mathcal{H} \subseteq \mathcal{F}$ a compact version $\mathcal{H}^{\prime}$ by taking the intersection of every member of $\mathcal{H}$ with an axis-aligned box $B . B$ is chosen large enough so that any feasible subfamily $\mathcal{G} \subseteq \mathcal{H}$ corresponds to a feasible subfamily $\mathcal{G}^{\prime} \subseteq \mathcal{H}^{\prime}$. Since the feasibility or infeasibility of subfamilies is preserved, the Helly number of $\mathcal{H}^{\prime}$ is the same as the Helly number of $\mathcal{H}$.

Theorem 3.1 implies that $\left(R^{d}, \mathcal{H}^{\prime}, \preceq\right)$ is a concrete LP-type problem for any $\mathcal{H}^{\prime}$, so, by Lemma 2.1 , the Helly number of any $\mathcal{H}^{\prime}$, and hence any $\mathcal{H}$, is at most $k(d+1)$. Since all of its finite subfamilies $\mathcal{H} \subseteq \mathcal{F}$ have Helly number $k(d+1)$, so does $\mathcal{F}$.

Note that the condition that the intersection of any subfamily can be expressed as the disjoint union of a fixed number of convex sets is a strong one. It is not true in general for families of disjoint unions of at most $k$ convex sets, for instance. An example of a family that does meet the condition is one in which every set $G$ is a set of at most $k$ closed balls with a common radius $\delta_{G}$, such that the distance between any two balls is no less than $\delta_{G}$.

\section{Acknowledgments}

The author thanks Jiři Matoušek for suggestions on the presentation, and an anonymous referee for several clarifications.

\section{References}

[C] K. L. Clarkson, Las Vegas algorithms for linear and integer programming when the dimension is small, Journal of the ACM 42 (1995), 488-499.

[DGK] L. Danzer, B. Grünbaum, and V. Klee, Helly's theorem and its relatives, in Convexity (Proceedings of the Symposia on Pure Mathematics, vol. 7), pp. 101-180. American Mathematical Society, Providence, RI, 1963.

[E] J. Eckhoff, Helly, Radon and Carathéody type theorems, in Handbook of Convex Geometry, P. M. Gruber and J. M. Wills, eds., Chapter 2.1. Elsevier, Amsterdam, 1993.

[GM] B. Grünbaum and T.S. Motzkin, On components in some families of sets, Proceedings of the American Mathematical Society 12 (1961), 607-613.

[HT] W. R. Hare and G. G. Thompson, Some generalized Helly-type theorems, Congressus Numerantium 67 (1988), 5-18.

[L] D. G. Larman, Helly-type properties of unions of convex sets, Mathematika 15 (1968), 53-59.

[MSW] J. Matoušek, M. Sharir, and E. Welzl, A subexponential bound for linear programming, Proceedings of the 8th Annual Symposium on Computational Geometry, 1992, pp. 1-8.

[M] H. C. Morris, Two Pigeon Hole Principles and Unions of Convexly Disjoint Sets, Ph.D. thesis, California Institute of Technology, Pasadena, CA, 1973.

[S] R. Seidel, Linear programming and convex hulls made easy, Proceedings of the 6th Annual Symposium on Computational Geometry, 1990, pp. 211-215.

[SW] M. Sharir and E. Welzl, A combinatorial bound for linear programming and related problems, Proceedings of the 9th Annual Symposium on Theoretical Aspects of Computer Science, pp. 569-579. Lecture Notes in Computer Science, vol. 577. Springer-Verlag, Berlin, 1992. 